\title{
Ueber das «harnstoffbildende» Ferment der Leber.
}

Von

\author{
Dr. Otto Loewi,
}

Assistent an der inneren Abtheilung des städtischen Krankenhauses zu Frankfurt a. M.

(Aus dem physiologisch-chemischen Institut zu Strassburg. Neue Folge Nr. 11.) (Der Redaction zugegangen am 18. Jull 1898.)

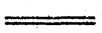

I.

Ch. Richet ${ }^{1}$ ) hat 1893 mitgetheilt, dass frisch entnommene, entblutete Hundeleber, wenn sie bei $39^{\circ}$ gehalten wird, eine Zunahme des Harnstoffgehalts erkennen lässt. Zur Harnstoffbestimmung bediente er sich dabei des Hypobromitverfahrens, wobei er, um das Ammoniak auszuschalten, die zur Analyse bestimmten Proben vor Zusatz der Bromlauge 24 Stunden bei alkalischer Reaction über Schwefelsäure stehen liess. Die bei diesen Versuchen beobachtete Vermehrung des durch Brom erhältlichen Stickstoffs wurde auf Harnstoffbildung bezogen, und Richet's Schüler Brief vermochte aus solcher «überlebenden» Leber kleine Mengen einer krystallographisch und chemisch als Harnstoff charakterisirten Substanz zu gewinnen.

Diese Angaben bedeuten im Hinblick auf die seit v. Schroeder festgestellte Lehre von der Harnstoffbildung in der Leber keinen wesentlichen Fortschritt. Da vielfach lebende Organe durch Blutentziehung das Functionsvermögen nur allmählich einbüssen, so konnte es sich auch hier um ein

1) Gh. Richet, Comptes rendus 118, 1125. 
allmähliches Versiegen der vitalen, harnstoffbildenden Function gehandelt haben.

Eine ganz andere Bedeutung beansprucht jedoch die kurz darauf erfolgte Mittheilung Richet's, ${ }^{1}$ ) wonach der wässerige, mit Fluornatrium vor Fäulniss geschützte und filtrirte Leberauszug und ebenso der daraus durch Alkohol gefällte Niederschlag die Fähigkeit der Harnstoffbildung besitzen sollte. Bei Untersuchung mit Bromlauge ergab sich nämlich im Leberauszuge direkt, und in noch höherem Maasse auf Zusatz des aus Leberextract dargestellten Alkoholniederschlages nach Digestion in Brutwärme eine Vermehrung des erhältlichen Stickstoffs. Bei Anwendung einer modificirten Hypobromitmethode, welche zugleich die Menge der oxydablen Stoffe zu bestimmen gestattet, konnte eine Vermehrung derselben in beträchtlichem Umfange sichergestellt werden. Darnach hält Richet die Annahme eines vom Protoplasma abtrennbaren, harnstoffbildenden Fermentes im Lebergewebe für gestattet.

Gottlieb ${ }^{2}$ ) fand bei Nachprüfung der Angaben Richet's im aseptisch bei $40^{\circ}$ digerirten Leberbrei eine Vermehrung eines in den Aetheralkohol übergehenden, stickstoffhaltigen Körpers, der durch Quecksilbernitrat, nicht aber durch Phosphorwolframsäure fällbar war und somit recht wohl Harnstoff sein konnte.

Ebenso fand vor Kurzem L. Schwarz ${ }^{3}$ ) eine Zunahme der Stickstoffwerthe, wenn er Leberbrei bei $40^{\circ}$ digerirte und darin nach Mörner-Sjö qvist's ${ }^{4}$ ) Harnstoffbestimmungsmethode - Ausziehen der mit Barytmischung gefällten Flüssigkeit mit Aetheralkohol, Entfernen des Ammoniaks mit Magnesia und Bestimmung des Stickstoffgehaltes — verfuhr. Die Bedeutung dieses Befundes wird von Schwarz durch die Bemerkung ins u. 525 .

1) ders., Comptes rendus de la société de biologie 1894. S. 368

2) Gottlieb, Münch. medic. Wochenschr. 1895 und Centralblatt f. d. Krankh. der Harnorgane 6. S. 480.

8) L. Schwarz. Arch. f. experim. Pathol. und Pharm. 41. S. 60.

4) Mörner-Sjöqvist, Scand. Arch. f. Physiologie, 1892. 
rechte Licht gesetzt, dass das Verfahren von MörnerSjöqvist strenggenommen nicht die Anwesenheit von Harnstoff, sondern nur eines von Ammoniak verschiedenen, ätheralkohollöslichen, stickstoffhaltigen Körpers beweise.

In einem neuen Licht erscheint Richet's Fermenttheorie in der etwa vor einem Jahr erschienenen Mittheilung von Chassevant und Richet, ${ }^{1}$ ) die sich mit der Frage beschäftigt, aus welchem Bestandtheil der Leber das fragliche Ferment Harnstoff bereitet. Aseptisch hergestellte Leberauszüge wurden mit Chloroform versetzt, filtrirt und zum Theil mit Ammoniumtartrat, zum Theil mit harnsaurem Natrium, zum Theil ohne Zusatz in Brutwärme digerirt. Allenthalben fand sich Zunahme des Harnstoffs; eine Abnahme der im Extract stets vorhandenen Eiweisskörper und des zugefügten Ammonsalzes war nicht nachweisbar, wohl aber des harnsauren Salzes. Chassevant und Richet schlossen daraus, dass die fermentative Bildung des Harnstoffs unter den gegebenen Bedingungen nicht (wenigstens nicht unmittelbar) auf Kosten von Ammoniaksalzen oder Eiweisskörpern erfolgte, sondern auf Kosten von krystallisirbaren, stickstoffhaltigen Substanzen, unter Anderen der Harnsäure.

Ueber die Methode der Harnstoffbestimmung machen Chassevant und Richet keine Angaben: Allem Anschein nach bedienten sie sich neuerlich des Hypobromitvertahrens.

In allerjüngster Zeit endlich hat Spitzer²) Leberbrei und Leberauszüge auch auf Ammonsalze und Leucin in ammoniakalischer Lösung einwirken lassen und den Nachweis des etwa gebildeten Harnstoffs nach v. Schröder's ${ }^{3}$ ) sehr zuverlässiger, aber für sehr geringe Mengen nicht mehr ausreichend empfindlicher Methode zu führen gesucht - stets ohne Erfolg. Dasselbe negative Ergebniss hatte er bei in gleicher Weise durchgeführter Nachprüfung der Versuche von Chasse-

1) Chassevant et Gh. Richet, Comptes rendus de la société de . biologie. 1897. S. 793.

2) W. Spitzer, Pflüger's Archiv 71, 60.

3) v. Schröder, Arch. für experim. Pathol. u. Pharm. 15, 364. 
vant und Richet mit harnsaurem Alkali zu verzeichnen. Er betrachtet die Bildung des Harnstoffs als eine direkt an das Ueberleben des Organs und seine histologische Integrität geknüpfte Leistung.

II. Nachweis der Bildung einer ätheralkohollöslichen, stickstoffhaltigen Substanz im Lebergewebe.

Zum Verständniss der mitzutheilenden Versuche sei bemerkt, dass dieselben fast sämmtlich im Wintersemester 1896/97, also vor Erscheinen der einschlägigen Untersuchungen von Chassevant und Richet, L. Schwarz und W. Spitzer ausgeführt wurden. Der ursprüngliche Ausgangspunkt war, wie bei Spitzer, die Vermuthung, dass das harnstoffbildende Ferment eine oxydative Synthese im Sinne Hofmeister's ${ }^{1}$ ) vermittelt. Bald jedoch ergab sich die Nothwendigkeit, zunächst die Existenz einer fermentativen Einwirkung überhaupt und die Bedingungen, unter denen sie nachweisbar wird, klarzustellen.

Zunächst seien die Versuche mit Lebergewebe angeführt. Die Versuchsanordnung war folgende:

Einem eben verbluteten Hund - auf den Fütterungszustand des Thieres war in diesen Versuchen- nicht geachtet worden - wird unter möglichster Wahrung der Asepsis die Leber entnommen. Ein gewogener Theil wird sofort in viel 97\% igem Alkohol fein zerrieben, nach 24 Stunden filtrirt, der Rückstand nochmals ebenso behandelt. Ein anderer gewogener Theil wird nach mehrstündiger Digestion bei Körpertemperatur der gleichen Behandlung unterzogen. Aus den vereinigten Filtraten jeder Portion wird der Alkohol bei einer $60^{\circ}$ nicht übersteigenden Temperatur verjagt, der Rückstand mit wenig Wasser aufgenommen und darin der Harnstoff nach MörnerSjöqvist bestimmt. Zur Hintanhaltung der Fäulniss wird in sämmtlichen Versuchen Fluornatrium in solcher Dosirung zugesetzt, dass die steril $\mathrm{zu}$ haltende Flüssigkeit eine 1\% ige Fluornatriumlösung vorstellt.

1) Hofmeister, ebenda 37, 426. 


\begin{tabular}{|c|c|c|c|c|}
\hline $\begin{array}{l}\text { Ver- } \\
\text { such. }\end{array}$ & $\begin{array}{l}\text { Digestions- } \\
\text { dauer. }\end{array}$ & \multicolumn{2}{|c|}{$\begin{array}{l}\text { gr. Stickstoff berechnet auf } 1000 \mathrm{gr} \text {. Lèber } \\
\text { in der nicht digerirten । in der digerirten } \\
\text { Portion. }\end{array}$} & $\begin{array}{l}\text { Stickstoff- } \\
\text { zunahme } \\
\text { in gr. }\end{array}$ \\
\hline I. & 8 Stunden & 0,3727 & 0,4661 & 0,0934 \\
\hline II. & 8 & 0,4666 & 0,4713 & 0,0047 \\
\hline III. & 12 & 0,6775 & 0,7050 & 0,0275 \\
\hline IV. & 24 & 0,4039 & 0,4170 & 0,0131 \\
\hline
\end{tabular}

Eine Bestimmung in aliquoten Theilen des 0,7\% Chlornatrium haltenden Wasserauszuges der Leber nach gleichem Verfahren ergab keine Stickstoffzunahme im digerirten Theil.

Diese Ergebnisse bestätigen die Angaben von Richet, Gottlieb und L. Schwarz. Will man nicht zu künstlichen Deutungen greifen, so muss man annehmen, dass die Digestion von Leber und Leberbrei zum Auftreten oder zur Vermehrung einer stickstoffhaltigen Substanz führt, welche nicht Ammoniak ist, mit Bromlauge Stickstoff entwickelt (Richet), sich in Aetheralkohol löst, durch Mercurinitrat, nicht aber durch Phosphorwolframsäure gefällt wird (Gottlieb) und bei der Harnstoffbestimmung nach Mörner-Sjö qvist sich wie Harnstoff verhält (L. Schwarz, Verf.).

Ich will die Substanz, die in den angeführten Eigenschaften sich etwa wie ein Säureamid verhält, vorläufig als die ätheralkohollösliche Substanz bezeichnen.

Auffällig ist, dass die Menge der bei Digestion auftretenden Vermehrung an ätheralkohollöslichem Stickstoff öfters so gering ist, dass sie fast noch innerhalb der Fehlergrenzen gelegen sein kann. In keinem Fall erreichte sie die Werthe, wie sie L. Schwarz angibt. In der Annahme, es könnten die grossen Schwankungen vielleicht in einem Verhältniss stehen zu dem jeweilig von der Fütterung des Thieres abhängigen Reichthum der Leber an Harnstoffbildnern, wurde folgender Versuch angestellt.

\section{Versuch V.}

Ein Hund wurde 3 Tage hungern gelassen, ein zweiter auf reichliche Kost gesetzt. Dann wurden beide Thiere getödtet und die Lebern wie oben verarbeitet. 
1000 gr. Leber des Hungerhundes enthielten:

vor der Digestion 0,7214 gr. ätheralkohollöslichen Stickstoff, nach Zunahme $\frac{0,7931 \text { " }}{0,0717 \mathrm{gr} .}$

1000 gr. Leber des Futterhundes enthielten:

vor der Digestion $0,7915 \mathrm{gr}$. ätheralkohollöslichen Stickstoff, nach $\stackrel{1,7021 \text {. }}{\text { Zunahme }}$

III. Versuche über Bildung der ätheralkohollöslichen Substanz durch Fermentlösung.

Das eben angeführte Resultat macht die Ungleichheiten verständlich, welche in Richet's und meinen Versuchen in Betreff der Menge der durch Lebergewebe gebildeten harnstoffähnlichen Substanz hervortraten. Zugleich weist es den. Weg zu einer erfolgreichen Untersuchung des vermutheten Fermentes.

Da man nicht von vornherein der Anwesenheit der für das Ferment angreifbaren Substanzen in Leberauszügen oder gar in gereinigten Fermentlösungen sicher sein kann, so war es nöthig, derartig angreifbare Stoffe ausfindig zu machen.

In dieser Richtung kamen die als Vorstufen des Harnstoffs anzusprechenden Substanzen zunächst in Betracht; also Ammoniak, sodann die als Verdauungsprodukte auftretenden Amidosäuren, deren leichte Ueberführbarkeit in Harnstoff im Organismus seit Nencki und Schultzen feststeht, sodann die dem Harnstoff nahestehenden Säureamide - Formamid und Oxaminsäure, eventuell auch die Harnsäure.

In Bezug auf Ammoniak und die beiden letztgenannten Stoffe liegen bereits Angaben vor:

L. Schwarz fand bei Digestion von Leberbrei die annähernd gleiche Zunahme an ätheralkohollöslichem Stickstoff, ob er denselben ohne Zusatz oder nach Hinzufügen von oxaminsaurem Natrium, Ammoniumcarbonat oder beiden zusammen - der Digestion unterwarf. Chassevant und Richet fanden den Zusatz von Ammoniumtartrat ohne Einfluss, nach Zugabe von harnsaurem Salz beobachteten sie erst 
nach 48 Stunden eine deutliche Zunahme des auf Harnstoff bezogenen Stickstoffs, sodass eine Spaltung der Harnsäure durch das fragliche Ferment, wie bereits Spitzer hervorhebt, wenig Wahrscheinlichkeit hat.

Für meine Versuche, die, um einer Täuschung durch Bildung von Ammoniak vorzubeugen und doch nicht etwa auftretende basische Zwischenprodukte auszuschliessen, mit dem Verfahren von Mörner-Sjöqvist ausgeführt wurden, kamen selbstverständlich nur. Körper in Betracht, von deren absoluter Unlöslichkeit in Aetheralkohol ich mich durch blinde Versuche vorher überzeugt hatte. Dabei erwies sich Glycocoll als besonders brauchbar. Hingegen waren dadurch Versuche mit Formamid, die sonst nahe gelegen hätten, ausgeschlossen.

Zunächst handelte es sich darum, zu zeigen, dass die Bildung der fraglichen Substanz nicht durch zellige Elemente oder Zellreste vermittelt wird.

Versuch VI.

Einige Cubikcentimeter concentrirten, wässerigen Leberextractes werden mit $0,058 \mathrm{gr}$. Glycocoll (Merck) und einigen Tropfen neutralen essigsauren Ammons versetzt. Das mit kohlensaurem Ammon schwach alkalisch gemachte Gemisch ergibt nach 14 stündiger Digestion bei $4000,0156 \mathrm{gr}$. ătheralkohollöslichen Stickstoff; die Kontrollprobe, die ohne Zusatz blieb, ergibt: $0,0015 \mathrm{gr}$.

Um die Mitwirkung zelliger Elemente mit Sicherheit auszuschliessen, trieb ich das zweimal durch Papier fiitrirte Extract durch ein Chamberland-Filter. Ich erhielt so eine schwach gelb gefärbte, absolut klare Lösung.

\section{Versuch VII.}

Der Gehalt der Fermentlösung an ätheralkohollöslichem Stickstoff, wie stets nach Mörner-Sjöqvist bestimmt, betrug 0,0013 gr., der einer aliquoten Portion, die nach Zusatz von 0,585 gr. Glycocoll einige Stunden bei $40^{\circ}$ gehalten wurde, $0,0102 \mathrm{gr}$. Also wiederum eine beträchtliche Zunahme.

Nach solchem Ausfall der Versuche gewann die Annahme, dass es sich bei der Bildung des stickstoffhaltigen Körpers thatsächlich um eine Fermentwirkung handle, nicht wenig an Wahrscheinlichkeit. Ich musste demnach die Wirksamkeit von «Fermentlösungen» aus Lebersubstanz prüfen. 
Zur Herstellung der Fermentlösung bediente ich mich des gewöhnlich geübten Verfahrens. Das Organ, Hunde- oder Rindsleber, wurde fein zerhackt, einige Stunden unter Zusatz von Fluornatrium und häufigem Schütteln mit physiologischer Kochsalzlösung behandelt und über Nacht stehen gelassen. Die wässerige Schicht wurde abgehebert und mit dem Vielfachen von 97\% igem Alkohol gefällt, filtrirt, der Rückstand lufttrocken gemacht, fein gepulvert, mit Wasser einige Stunden bei Zimmertemperatur stehen gelassen und filtrirt. Vor dem Versuch wurde die Lösung durch kohlensaures Natrium schwach alkalisch gemacht und mit Fluornatrium in obengenannter Dosirung versetzt. In den ersten Versuchen wurde jedesmal dem stickstoffhaltigen Körper Ammoniak zugesetzt, um Material für die etwaige Bildung der zweiten Amid-Gruppe in Harnstoff beizustellen. Es stellte sich aber heraus, dass auch ohne diesen Zusatz die digerirte Portion ein Plus an ätheralkohollöslichem Stickstoff zeigte. Die verwendeten Präparate stammten ausnahmslos von Merck. Bei sämmtlichen Versuchen wurden Kontrollanalysen ausgeführt. Die nachstehend mitgetheilten Stickstoffzahlen geben sämmtlich die nach Mörner-Sjöqvist ermittelte Menge des ätheralkohollöslichen Stickstoffs an.

Versuch VIII. Digestionsdauer 4 Stunden.

$5 \mathrm{ccm}$. Fermentlösung ohne Zusatz $=0$ gr. $\mathrm{N}$,

$5 \gg \quad$ mit Zusatz von 0,012 gr. Ammoniumacetat $=0$ gr. N,

$5 \gg>>\quad>022$ * Glycocoll =0,0041 gr. N,

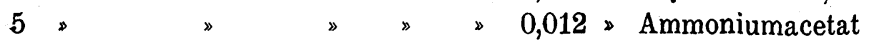

$+0,022$ gr. Glycocoll

$=0,0044$ gr. $\mathrm{N}$,

5 . " " $\quad 0,012$, Ammoniumacetat $+0,2 \mathrm{gr}$. Glycocoll $=0,0081 \mathrm{gr}$. N.

Versuch IX. Digestionsdauer 4 Stunden.

$5 \mathrm{ccm}$. Fermentlösung ohne Zusatz $=0,0024 \mathrm{gr}$. N,

\begin{tabular}{|c|c|c|c|c|c|c|}
\hline 5 & s & ه & mit & Zusatz von & n 0,012 & $\triangle$ Ammoniumacetat \\
\hline 5 & " & > & " & 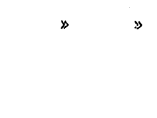 & 0,012 & $\begin{array}{l}\text { Ammoniumacetat } \\
+0,5 \text { gr. Glycocoll } \\
=0,0123 \text { gr. } \mathrm{N},\end{array}$ \\
\hline
\end{tabular}

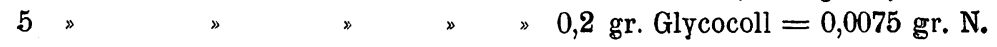


Diese Versuche sind aus einer grossen Zahl, im Ergebniss gleichlautender, herausgegriffen. Sie zeigen zur Evidenz, dass sich auch unter dem Einfluss der "Fermentlösung 》 aus dem ätheralkoholunlöslichen Glycocoll bei der Digestion eine ätheralkohollösliche Substanz bildet, gleichviel ob daneben ein Ammoniaksalz zur Verfügung steht oder nicht. Für den Fall, dass der gebildete Körper Harnstoff ist, müsste demnach angenommen werden, dass 2 Moleküle Glycocoll ihre Amidgruppen beistellen. Ganz negativ war der Ausfall der Digestion mit essigsaurem Ammon allein.

Dass es sich in den vorgenannten Versuchen um eine Wirkung handelt, die nach Erhitzen auf $100^{\circ}$ ausbleibt, lehren die folgenden Zahlen:

\section{Versuch X.}

$5 \mathrm{ccm}$. Fermentlösung ohne Zusatz 4 Stunden digerirt $=0,0025 \mathrm{gr} . \mathrm{N}$, 5 . mit Zusatz von Glycocoll $=0,0078$ gr. N,

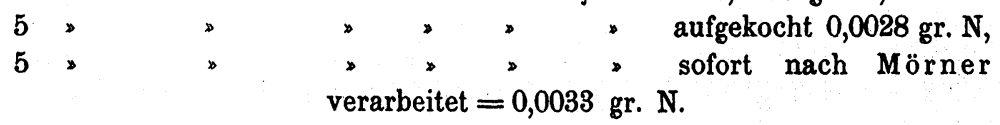

Die letzte Zahl zeigt zugleich, dass unter dem Einfluss der Fermentlösung die Lösungsverhältnisse des Glycocolls in Aetheralkohol nicht merklich alterirt werden.

Der Vollständigkeit halber seien noch einige Versuche mit anderen stickstofthaltigen Körpern angefügt.

Versuch XI.

Digestionsdauer 5 Stunden.

$10 \mathrm{ccm}$. Fermentlösung ohne Zusatz $=0,0025 \mathrm{gr} . \mathrm{N}$,

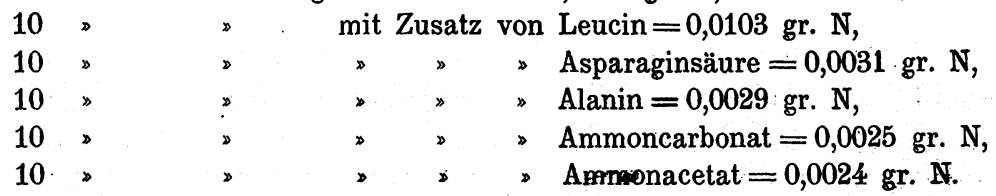

Schon diese kleine Reihe zeigt, dass das Ferment nur. auf einen Theil der Stoffe Einfluss übt, die der Organismus leicht und vollständig in Harnstoff überführt. Da schon nach dem. Gesagten feststand, dass der zellenfreie Leberauszug aus Amidosäuren (Glycocoll, Leucin) eine ätheralkohollösliche, stickstoffhaltige Substanz bildete, nicht aber aus Ammonsalzen, 
nahm ich zunächst von einer systematischen Durchprüfung Abstand, auf welche Harnstoffbildner das Ferment sich wirksam erweist, und wandte mich der Frage $\mathrm{zu}$, ob die entstandene, ätheralkohollösliche Substanz Harnstoff ist oder nicht.

\section{Ist die durch die Fermentlösung aus Amidosäuren gebildete Substanz Harnstoff?}

Die Beantwortung dieser Frage war einerseits durch den Umstand sehr erleichtert, dass nicht mit Leberbrei oder Leberextracten, die ja Reste des in der Leber präformirten Harnstoffs enthalten können, sondern mit durch Alkoholfällung bereiteten, von Harnstoff sicher freien Fermentlösungen gearbeitet werden konnte. Von grossem Vortheil war ferner, dass man bei Anwendung geeigneten, angreifbaren Materials - ich wählte dazu, als bestuntersuchtes, Glycocoll - auf sicheren Erfolg der Einwirkung rechnen kann und von den Zufälligkeiten, die den Verlauf des Versuchs bei Benutzung von Leberbrei und Leberextracten beeinflussen, gänzlich unabhängig blieb.

Zur Darstellung ausreichender Mengen der fraglichen Substanz verfuhr ich wie in den bis jetzt geschilderten Versuchen, nur dass ich grössere Mengen von Fermentlösung und Zusatz (Glycocoll) in Arbeit nahm. Nach der Digestion wurde mit Aetheralkohol und Barytmischung in dem von MörnerSjöqvist angegebenen Verhältniss gefällt; in das Filtrat übergegangene Spuren von Baryt wurden durch Einleiten von Kohlensäure und nochmalige Filtration entfernt, das Filtrat nach Zusatz von Magnesia bei einer $60^{\circ}$ nicht übersteigenden Temperatur zur Trockne gebracht, noch einige Male mit Aetheralkohol aufgenommen, filtrirt und eindunsten gelassen. Es hinterblieb ein syrupöser Rückstand, bräunlich gefärbt, der nach einigem Stehen theilweise auskrystallisirte. Der Versuch, in diesem Auszug Harnstoff mit Hülfe 'der Salpetersäureprobe oder nach Lüdy ${ }^{1}$ ) nachzuweisen, schlug stets fehl. Da letztere Probe, wenn sie negativ ausfällt, die Gegenwart auch der kleinsten Mengen Harnstoff ausschliesst, erscheint durch das

1) Lüdy, Monatshefte für Chemie. 10, 310. 
angeführte Ergebniss die Annahme einer fermentativen Bildung von Harnstoff aus Glycocoll ausgeschlossen.

Betreffs der die Stickstoffvermehrung bedingenden, ätheralkohollöslichen Substanz konnte in Anbetracht der geringen Ausbeute nur ermittelt werden, dass sie mit fixem Alkali reichlich Ammoniak abspaltet. Der Versuch, in dem Rückstand des Aetheralkoholauszugs die Gegenwart von Blausäure oder Cyanursäure nachzuweisen, verlief negativ. Die naheliegende Vermuthung, dass es sich um Formamid handle, schien sich bei einem Versuch zu bestätigen, wo es gelang, in dem Rückstand Ameisensäure nachzuweisen. Doch war dieser Befund nicht constant, und der Versuch, Formamid nach der allerdings nicht sehr empfindlichen, aber zuverlässigen Methode von Cleve und Halsey ${ }^{1}$ ) in Form der charakteristischen Chinaldinverbindung zu isoliren, war ohne Erfolg.

\section{Ergebnisse.}

Die vorgeführten Versuche über das harnstoffbildende Ferment lehren, dass eine fermentative Harnstoffbildung aus Glycocoll (und wohl auch anderen Amidosäuren) im Sinne Richet's nicht besteht, wohl aber die fermentative Bildung einer stickstoffhaltigen Substanz, die sich in vieler Beziehung wie Harnstoff verhält und demgemäss das positive Ergebniss der Versuche von Richet, Gottlieb und L. Schwarz erklärt. Damit entfallen denn auch alle Schwierigkeiten, die sich der Annahme eines harnstoffbildenden Fermentes auf Grund der gangbaren Vorstellungen über Fermentwirkung entgegenstellen.

Die synthetische Bildung des Harnstoffs aus seinen Vorstufen hat sich somit ebensowenig von der lebenden Zelle getrennt nachahmen lassen, als das bei anderen vitalen Synthesen - der Hippursäurebildung (Schmiedeberg-Bunge) und der Methylsynthese (Hofmeister) - gelungen ist.

Hingegen beansprucht die Thatsache, dass die sonst selbst. durch concentrirte Säuren und Alkalien chemisch kaum angreifbaren Amidosäuren einfach durch ein fermentartiges Agens

1) Halsey, Zeitschr. f. physiolog. Chemie. XXV, S. 330 . 
in eine Verbindung, die locker gebundenen Stickstoff hält, übergeht, physiologisches Interesse. Der Organismus gebietet damit über ein Mittel, fest gebundene Amidogruppen, wie sie vorwiegend im Eiweissmolekül enthalten sind und die sonst selbst durch concentrirte Säuren und Alkalien nicht abgespalten werden können, in locker gebundene überzuführen und so der Harnstoffbildung zugänglich $\mathrm{zu}$ machen.

In einer jüngst erschienenen Arbeit hat Salaskin ${ }^{1}$ ) den Nachweis geführt, dass die Durchblutung der Leber unter Zusatz von Glycocoll, Leucin und Asparaginsäure zu einer Erhöhung der (nach Schöndorff) ermittelten Harnstoffwerthe führt. Da das Schöndorff'sche Verfahren nicht direkt den Harnstoff, sondern streng genommen nur den aus den Amidosäuren entstandenen, locker gebundenen, durch Säuren abspaltbaren Stickstoff bestimmt, so bleibt die Möglichkeit offen, dass Salaskin nicht den Harnstoff als solchen, sondern die von mir beobachtete, fermentativ aus den Amidosäuren gebildete, stickstoffhaltige Substanz ermittelt hat. Vom physiologischen Gesichtspunkt aus kann man diesem Einwurf keine principielle Bedeutung beimessen, weil der Uebergang einer solchen Substanz mit locker gebundenem Stickstoff in Harnstoff aller Wahrscheinlichkeit nach mindestens ebenso leicht exfolgt, wie die entsprechende Umwandlung der Ammonsalze, das physiologische Endergebniss somit das gleiche bleibt.

Für das Verständniss des bei der Harnstoffbildung sich abspielenden chemischen Vorganges ist jedoch der Nachweis eines fermentativ entstandenen Zwischengliedes zwischen Amidosäuren und Harnstoff von wesentlicher Bedeutung, denn er lehrt, dass eine vollständige Zertrümmerung der das Eiweiss zusammensetzenden Complexe mit fest gebundenem Stickstoff bis zur Bildung von Kohlensäure und Ammoniak nicht die nothwendige Vorbedingung der Harnstoffsynthese vorstellt, sondern dass diese auch auf anderem Wege unter Umwandlung der fest gebundenen Amidogruppe in eine locker gebundene erfolgen kann.

1) Sergej Salaskin, Zeitschrift f. physiol. Chemie. XXV, S. 128. 